\title{
Balanced Antipodal Vivaldi Antenna for Microwave Tomography
}

\author{
Syed Ahsan, Bright Yeboah-Akowuah, P Kosmas \\ School of Natural and Mathematical Sciences \\ King's College London, Strand WC2R 2LS \\ London, UK
}

\begin{abstract}
Balanced Antipodal Vivaldi Antennas (BAVAs) have been recently proposed for ultra-wideband (UWB) microwave imaging in medical applications such as breast cancer detection. This paper presents a modified design that allows the use of wideband BAVAs in microwave tomography, where the frequency range of interest is significantly lower than the UWB range down to $1 \mathrm{GHz}$. The performance of the antenna is evaluated using full-wave electromagnetic simulations.
\end{abstract}

Keywords-component; Microwave Imaging; Wideband Antenna; Cancer Detection; vivaldi antenna

\section{INTRODUCTION}

Near-field microwave imaging (MWI) methodologies for exploring the inner structure of the human body have been of growing interest recently. For example, microwave based approaches have been employed for the heart, bones and breast imaging [1]-[3], where the dielectric properties of breast tissues at microwave frequencies is identified as the key source of motivation behind MWI techniques [4]. In breast cancer applications, several research papers [5]-[14] outline the fact that the MWI is a low cost, nonionizing and quantitative (3-D) imaging modality that is promising not only for early detection of tumours, but also for monitoring the changes occurring in the breast tissues. Beamforming MWI approaches use a short time pulse at ultra-wideband (UWB) frequencies to illuminate the object under study (e.g. breast) using one or multiple antennas, and the reflected signals are then used to construct an image that locates areas of strong backscattered signal [9]. Microwave tomography uses lower frequencies to reconstruct the imaging domain of interest via a solution to the inverse scattering problem, resulting in a spatial distribution of the dielectric properties of material throughout the volume of the object (breast in the case of breast imaging)

An important aspect of all MWI approaches is the use of a suitable antenna element or array, which can obtain reliable sensing of the signals reflected from and transmitted through the interrogated tissue. Suitable antenna candidate should be characteristically compact for ease of placement around the breast, and must also be compatible with the imaging prototype configuration [15]. In microwave tomography, the choice of antenna will dictate the range of employed frequencies and the number of elements in the setup, which in turn affect the ill-posedness of the inverse scattering problem,

\author{
Helena Cano García, George Palikaras, Efthymios \\ Kallos \\ MediWise, Medical Wireless Sensing Ltd \\ 42 New Road, E1 2AX \\ London, UK
}

the computational efficiency of the imaging algorithm, and thus the quality of the resulting images

The Balanced Antipodal Vivaldi Antenna has been introduced in [16] for UWB breast imaging, and has been shown to exhibit low reflections from the feed and a compact profile. BAVAs feature end-fire pattern and can be made more directive by introducing an additional piece of higher permittivity element (Director) in the antenna aperture [16]. This director enables the BAVA to focus the radiated energy in the end-fire direction in the near-field region.

The BAVA designs proposed in [16] operate in the UWB frequency range (3-10 GHz), which is not useful in microwave tomography due to low penetration depth and the increased illposedness of the inverse scattering problem in the UWB frequency range. Thus the purpose of this paper is to introduce a novel BAVA design that can accommodate the lower frequency range used in microwave tomography (down to 1 $\mathrm{GHz}$ ), and at the same time retain the attractive features of the BAVA design. The proposed design criteria include good focusing in the near field and wideband operation, which allows the advantageous use of multiple-frequencies in the reconstruction process [13].

\section{DESIGN DETAILS}

\section{A. BAVA Geometry}

BAVA under study is depicted in Figure 1, where the top view of the antenna is shown. It consists of three metal components and two substrate layers, two metal components serve as ground planes while the middle patch is the active conducting component. The dimensions for this design are 75 $\mathrm{mm} * 100 \mathrm{~mm} * 3 \mathrm{~mm}$, plus the ground and patch thickness which has been kept at $0.035 \mathrm{~mm}$. The antenna is fed through an SMA connector which is followed by a gradual transition from stripline to tristripline. The conductor width increases linearly in the transition region, whereas the ground width decreases exponentially. This results in constant impedance. The tristrip is extended for a short distance, after which the ground and conductor start to flare exponentially in opposite directions resulting in antenna aperture. 


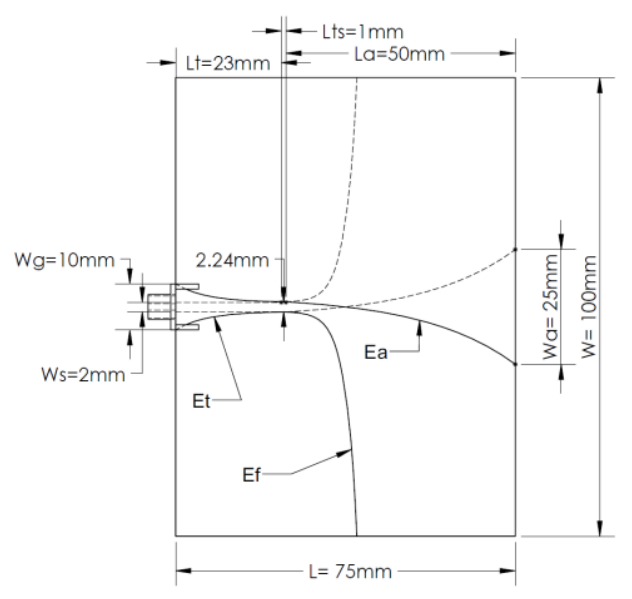

Figure 1: BAVA Geometry

\section{B. Materials and Parameters}

The proposed BAVA has two substrate layers of (Rogers Corporation, CST, USA) RT/duroid 6002 with relative permittivity 2.94 , the metallic components are annealed copper and the antenna under study is excited by a coaxial waveguide port with SMA connector. The material chosen for the director of BAVA is Rogers TMM6 with relative permittivity equal to 6 . The ground plane and patch have same profile curves but in opposite direction with respect to each other. The ground planes are exposed whereas the patch is sandwiched between two substrate layers. The exponential equations are given below:

$$
Z= \pm A * e^{P *(x-B)}+C
$$

The design parameters of the geometry of BAVA under study are given in table 1.The parameters can be classified into separate categories based upon their functionalities; L and $\mathrm{W}$ are the length and width parameters, the aperture parameters include aperture length $\mathrm{La}=50 \mathrm{~mm}$ and aperture width $\mathrm{Wa}=26$ $\mathrm{mm}$. The transition parameters comprise of ground width of stripline $\mathrm{Wg}=10 \mathrm{~mm}$, width of tristrip Wts $=2.24 \mathrm{~mm}$, strip width of stripline $\mathrm{Ws}_{\mathrm{s}}=2 \mathrm{~mm}$, transition length $\mathrm{Lt}=23 \mathrm{~mm}$, the tristrip length Lts $=1 \mathrm{~mm}$. $\mathrm{Pt}=-0.15$ is the magnification factor for the transition curve, $\mathrm{Pf}=0.4$ is the magnification factor for the flare curve and $\mathrm{Pa}=0.05$ is the magnification factor for the aperture curve. Af, Aa and At are the scaling factors for the flare, aperture and transition curves respectively, with their parametric relations given in Table 1 .

Table1: Design Parameters of BAVA

\begin{tabular}{|c|c|c|c|c|}
\hline Curve & $\mathrm{A}$ & $\mathrm{P}$ & $\mathrm{B}$ & $\mathrm{C}$ \\
\hline$E t$ & $\frac{W t s-W g}{2 *(\exp (P t * L t)-1)}$ & $P t$ & 0 & $\frac{W g}{2}-A t$ \\
\hline$E f$ & $A f$ & $P f$ & $\begin{array}{l}L t \\
+L t s\end{array}$ & $\frac{W t s}{2}-A f$ \\
\hline$E a$ & $\frac{W t s+W a}{2 *(\exp (P a * L a)-1)}$ & $P a$ & $\begin{array}{l}L t \\
+L t s\end{array}$ & $\begin{array}{l}-\frac{W t s}{2} \\
-A a\end{array}$ \\
\hline
\end{tabular}

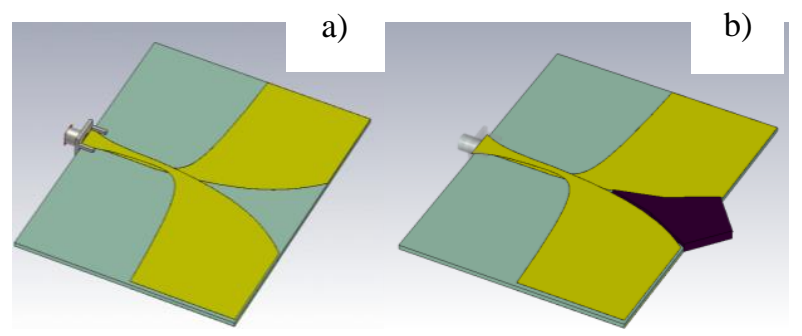

Figure 2. a) BAVA b) BAVA-D

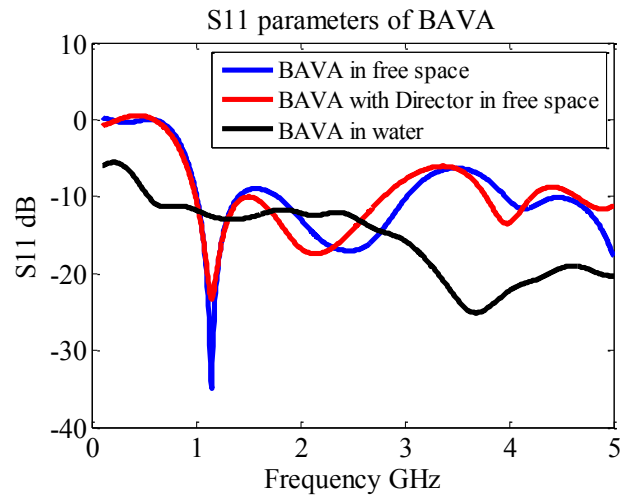

Figure 3: $\mathrm{S}_{11}$ parameters of BAVA, BAVA-D in free space and BAVA in water

\section{RESULTS}

The antenna is designed and simulated in $\mathrm{CST}^{\mathrm{TM}}$ Microwave Studio. The layouts of BAVA and BAVA-D are shown in figure 2.

\section{A. Return Loss}

As argued in the introduction, this antenna has been designed to operate in the microwave tomography frequency range from 0.5 to $4 \mathrm{GHz}$. The cutoff frequency for BAVA in free space is $0.99 \mathrm{GHz}$ where reflection below $-10 \mathrm{~dB}$ is achieved. The BAVA with a director in free space as well as BAVA immersed in water have also been simulated and their respective $S_{11}$ parameters are depicted in Figure 3. For the BAVA immersed in water, the first resonance occurs at 0.5 $\mathrm{GHz}$. All three antennas exhibit wideband behavior and can be used in the desired frequency range. Water as an immersion has been considered just to observe what effect an immersion medium will bring on the resonance frequency of the antenna.

\section{B. NearField Plots}

Nearfield radiation is of critical importance in MWI, and thus electric and magnetic field plots of the proposed BAVA and BAVA-D are given below. Figure $4 \mathrm{a}$ and $4 \mathrm{~b}$ depict the electric field plots for the BAVA and BAVA-D designs in free space for the $\mathrm{X}-\mathrm{z}$ plane in Figure 2. The depicted nearfield plots correspond to a frequency of $1.1 \mathrm{GHz}$, which is within the range most commonly used in microwave tomography. Similarly magnetic field plots for BAVA and BAVA-D in free space are depicted in Figures $4 \mathrm{c}$ and $4 \mathrm{~d}$. The electric field radiation is not very focused at $1.1 \mathrm{GHz}$, however a visible difference between the focusing in the presence and the absence of director can be observed. The focusing improves as 
the frequency increases, which is primarily due to the fact that the end-fire pattern doesn't exist at the lower frequencies, but as the frequency is increased a better radiation in the end-fire pattern is achieved. The dielectric loading attenuates the fields due to the dielectric losses, dielectric such as water (high permittivity) has shown substantial amount of loss. a)
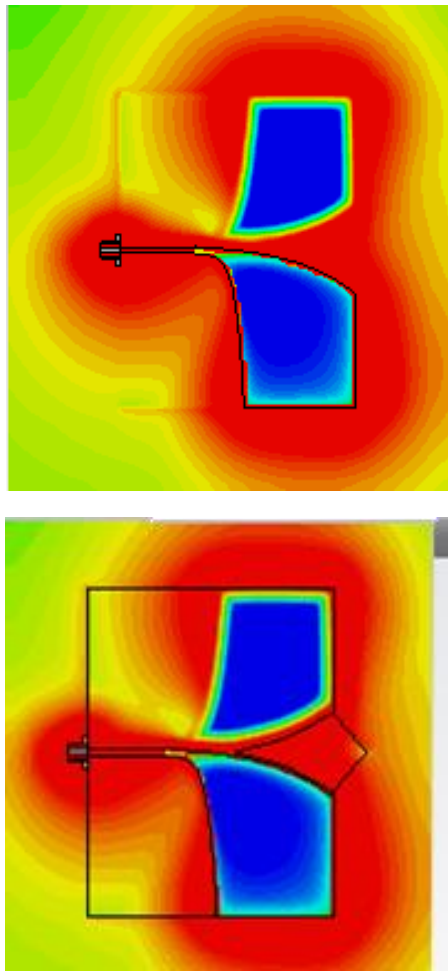

c)
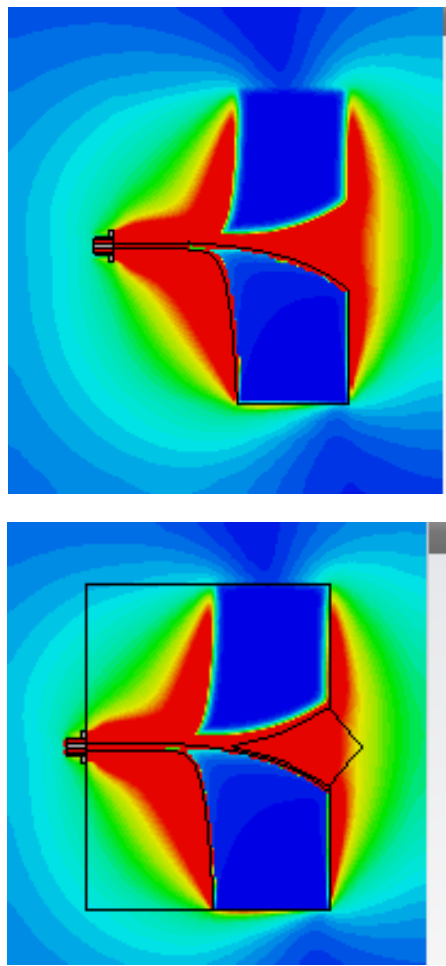

$d B(1 \% / m)$

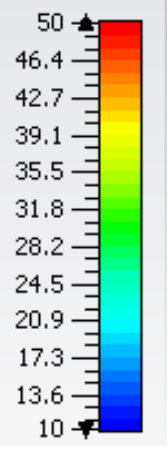

$d B(1 \% / m)$

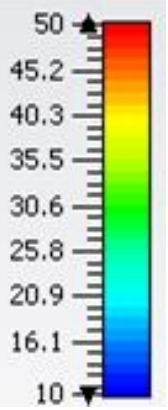

$A / \mathrm{m}$

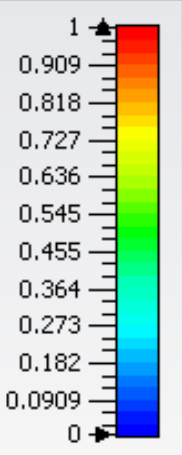

\section{$\mathrm{A} / \mathrm{m}$}

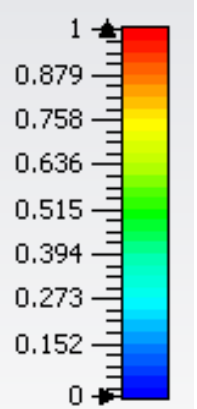

Figure 4: The near field plots: a) Electric field of BAVA, b) Electric field of BAVA-D, c) Magnetic field of BAVA, d) Magnetic field of BAVA-D

\section{Farfield Directivity Abs (Theta $=90)$}

a)

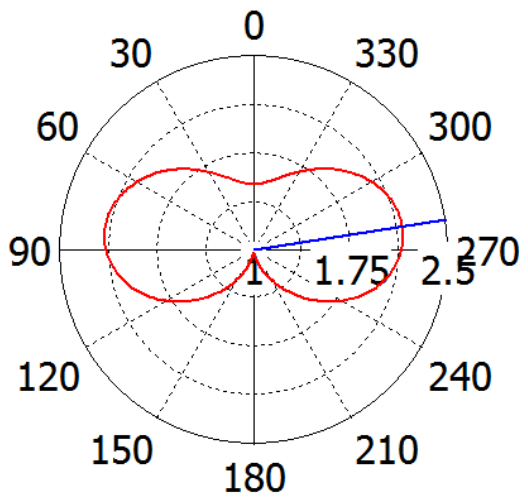

Phi / Degree vs. dBi

\section{Farfield Directivity Abs (Theta $=90$ )}

b)

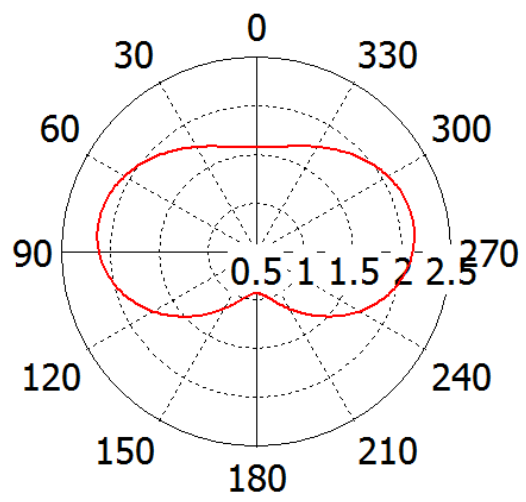

Phi / Degree vs. dBi

Figure 5: Farfield plots at $1.13 \mathrm{GHz}$ a) BAVA b) BAVA-D

\section{FarField Plots}

The far-field radiation patterns of the BAVA and BAVA-D are plotted on the E-plane in Figure 5a and 5b. It is observed that at low frequencies the director effect on antenna directivity is not profound; however, at higher frequencies the, the director has a substantial effect on the far-field radiation and half energy beam width of the antenna.

\section{CONCLUSION}

A BAVA with and without a director element has been designed for use in microwave tomography, and the performance has been analysed through results for the lower frequencies of operation. The proposed design considered a narrower aperture width and a broader total width along with shorter total length, resulting in a $S_{11}$ of $-10 \mathrm{~dB}$ or lower for 
frequencies higher than $500 \mathrm{MHz}$. As expected, the inclusion of an immersion medium lowers the frequency of operation further at the expense of increasing undesirable losses to the power delivered by the antenna to the imaging domain. We have also observed that the effect of the diamond shape director of higher permittivity material is not very profound at lower frequencies, but results in higher directivity as the frequency increases. Future work will focus on the modifications of this design to reduce its overall dimensions for use in a multi-antenna array.

\section{REFERENCES}

[1] S. Semenov, V. Posukh, A. Bulyshev, and T.Williams, "Microwave tomographic imaging of the heart in intact swine," J. Electromagn.Waves Applicat., vol. 20, no. 7, pp. 873-890, 2006.

[2] S. Semenov et al., "Microwave tomography for functional imaging of extremity soft tissues: Feasibility assessment," Phys. Med. Biol., vol.52, no. 18, pp. 5705-5719, 2007.

[3] T. Rubaek, P. Meincke, and O. Kim, "Three-dimensional microwave imaging for breast-cancer detection using the log-phase formulation," in Proc. IEEE Antennas Propag. Soc. Int. Symp., 2007, pp. 2184-2187.

[4] J. E. Joy, E. E. Penhoet, and D. B. Petitti, Eds., Saving Women's Lives: Strategies for Improving Breast Cancer Detection and Diagnosis Washington, DC, USA: National Academies Press, 2005, Institute of Medicine.

[5] P. M. Meaney, M. W. Fanning, T. Raynolds, C. J. Fox, Q. Q. Fang, C. A. Kogel, S. P. Poplack, and K. D. Paulsen, Initial clinical experience with microwave breast imaging in women with normal mammography," Acad. Radiol., vol. 14, no. 2, pp. 207-218, Feb. 2007.

[6] S. P. Poplack, T.D. Tosteson, W. A.Wells, B. W. Pogue, P. M.Meaney, A. Hartov, C. A. Kogel, S. K. Soho, J. J. Gibson, and K. D. Paulsen, "Electromagnetic breast imaging: Results of a pilot study in women with abnormal mammograms," Radiology, vol. 243, pp. 350-359,May 2007.
[7] D. W. Winters, J. D. Shea, P. Kosmas, B. D. Van Veen, and S. C. Hagness, "Three-dimensional microwave breast imaging: Dispersive dielectric properties estimation using patient-specific basis functions," IEEE Trans. Med. Imag., vol. 28, no. 7, pp. 969-981, Jul. 2009.

[8] T. Rubaek, O. S. Kim, and P. Meincke, "Computational validation of a 3D microwave imaging system for breast cancer screening," IEEE Trans. Antennas Propag., vol. 57, no. 7, pp. 2105-2115, Jul. 2009.

[9] P. Kosmas and C. M. Rappaport, "A matched-filter FDTD-based time reversal approach for microwave breast cancer detection" IEEE Trans. Antennas Propag., vol. 54, no. 4, pp. 1257-1264, Apr 2006.

[10] J. E. Johnson, T. Takenaka, K. Ping, S. Honda, and T. Tanaka, "Advances in the 3-D forward-backward time-stepping (FBTS) inverse scattering technique for breast cancer detection," IEEE Trans. Biomed. Eng., vol. 56, no. 9, pp. 2232-2243, Sep. 2009.

[11] N. Irishina, D. Álvarez, O. Dorn, and M. Moscoso, "Structural level set inversion for microwave breast screening," Inv. Probl., vol. 26, 035015 (26 pp.), Feb. 2010.

[12] J. D. Shea, P. Kosmas, S. C. Hagness, and B. D. Van Veen, "Contrast enhanced microwave imaging of breast tumors: A computational study using 3-D realistic numerical phantoms," Inv. Probl., vol. 26, 074009 (22 pp.), Jun. 2010.

[13] J. D. Shea, P. Kosmas, S. C. Hagness, and B. D. Van Veen, "Threedimensional microwave imaging of realistic numerical breast phantoms via a multiple-frequency inverse scattering technique," Med. Phys., vol. 37, no. 8, pp. 4210-4226, Aug. 2010.

[14] T. M. Grzegorczyk, P. M. Meaney, P. A. Kaufman, R. M. di FlorioAlexander, and K. D. Paulsen, "Fast 3-D tomographic microwave imaging for breast cancer detection," IEEE Trans. Med. Imag., vol. 31, no. 8, pp. 1584-1592, Aug. 2012.

[15] P. M. Meaney, M. W. Fanning, D. Li, S. P. Poplack, and K. D. Paulsen, "A Clinical Prototype for Active Microwave Imaging of the Breast," IEEE Trans. Microwave Theory Tech., vol. 48, no. 11, Nov. 2000

[16] Jeremie Bourqui, Michal Okoniewski, Elise C. Fear "Balanced Antipodal Vivaldi Antenna for breast cancer detection," Proc. EuCAP 2007, Edinburgh, Nov 2007, pp. 1-5. 